\title{
Anthropogenic control on riverine organic carbon dynamics in the Changiiang River basin post-Three Gorges Dam period
}

\author{
Sugandha Panwar, Bi Lei, Shouye Yang \\ State Key Laboratory of Marine Geology, Tongji \\ University, Shanghai 200092, China
}

The Changjiang River originating from the Qinghai-Tibetan Plateau is the largest and longest river in Asia. The river carries approximately 480 million tons of sediment flux annually and hence is an important entity in the global carbon cycle. The enhanced anthropogenic perturbation in the form of dam constructions in the upper part of the basin is reported to influence the natural carbon budget. Following the impoundment of the Three Gorges Dam in the year 2003 frequent instances of droughts were prevalent in the middle and lower Changjiang basin. The one-year continuous sampling (year 2008-2009) carried at Nantong in Jiangsu province in China displays an interactive control of dam regulations and drought conditions. The results highlight the changes in the suspended sediment size, particulate organic carbon (POC), particulate nitrogen (PN) flux, and source of organic and inorganic carbon and functional relationship between the terrestrial and aquatic system in the changed environmental conditions. The prevalence of low POC $\%$ and PN\% associated with coarser sediments transported by high discharge and existence of a non-linear relationship between the organic carbon flux and suspended sediment concentration reveals the extraordinary pattern controlled by human-induced modifications. The findings specify the impact of large dams on the hydrological processes and global climate and contribute to developing new data inventory for understanding river dynamics in the fast changing world. 